\title{
Secretary-Treasurer
}

Jin Woo Chang, Seoul, Korea

Vice Secretary-Treasurer

Konstantin Slavin, Chicago, USA

$2^{\text {nd }}$ Vice Secretary-Treasurer

Osvaldo Vilela Filho, Goiânia, Brazil

\section{Past President}

Takaomi Taira, Tokyo, Japan

\section{Historians}

Philip L. Gildenberg, Houston, USA

Giovanni Broggi, Milano, Italy

\section{Board of Directors}

Ron Alterman, Boston, USA

Tipu Aziz, Oxford, UK

Jocelyne Bloch, Lausanne, Switzerland

Patric Blomstedt, Umeå, Sweden

Youssef G. Comair, Beirut, Lebanon

G. Rees Cosgrove, Boston, USA

Paresh Doshi, Mumbai, India

Jairo Espinoza, Bogota, Columbia

Marwan Hariz, London, UK

Mojgan Hodaie, Toronto, Canada

Christopher R. Honey, Vancouver, Canada

Michael Kaplitt, New York, USA

Zelma Kiss, Calgary, Canada

Kendall Lee, Rochester, USA

Joseph Neimat, Nashville, USA

Jean Regis, Marseille, France

Ali Rezai, Columbus, USA

Rick Schuurman, Amsterdam, NL

Vladimir Shabalov, Moscow, Russia

Byung-chul Son, Seoul, Korea

Roberto Spiegelmann, Tel Hashomer, Israel

Hiroki Toda, Osaka, Japan

Jürgen Voges, Magdeburg, Germany

Continental Vice Presidents

\section{North America}

Douglas Kondziolka, New York, USA

Central \& South America

Fabian Piedimonte, Buenos Aires, Argentina

Europe

Bart Nuttin, Leuven, Belgium

Asia

Bomin Sun, Shanghai, China

Africa/Near Middle East

Graham Fieggen, Cape Town, South Africa

Oceania

Terry Coyne, Queensland, Australia

Web Master

Clement Hamani, Toronto, Canada

\section{Newsletter Editor}

Erich Richter, New Orleans, USA

\section{Membership}

Jason M. Schwalb, West Bloomfield, USA

\section{Journal Editor}

David W. Roberts, Lebanon, USA

\section{WSSFN Committee Heads}

Psychiatric Neurosurgery: Bart Nuttin, Leuven, Belgium Education: Joachim K. Krauss, Hannover, Germany Scientific Research: Andres Lozano, Toronto, Canada Industrial Relations: Joseph Neimat, Nashville, USA

WSSFN Scientific Program Chairs

Mojgan Hodaie, Toronto, Canada

Jean Regis, Marseille, France

\section{World Society for Stereotactic and Functional Neurosurgery BERLII , GERMANY}

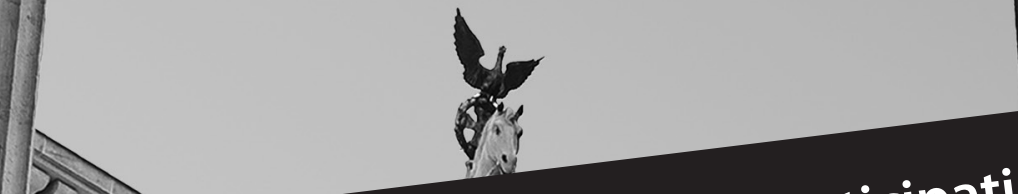

Thank you to all those participating

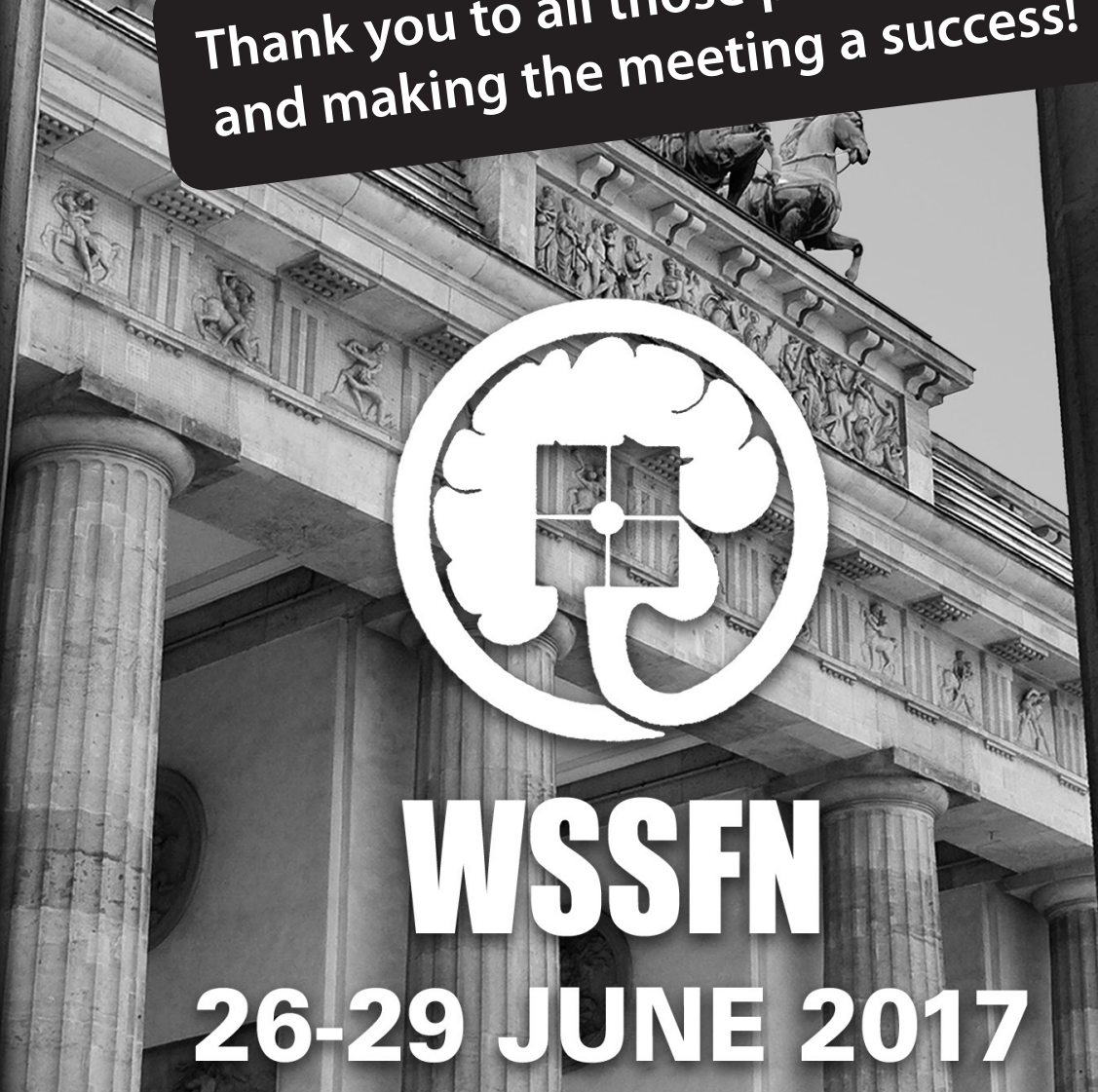

INTERCONTINENTAL BERLIN

$17^{\text {th }}$ Quadrennial Meeting EMERGING TECHNIQUES AND INDICATIONS

www.wssfin.org www.wssfn-congress.org 